\title{
Link Aware Data Aggregation Mechanism based on Passive Clustering in Wireless Sensor Network
}

\author{
K. Sruthi ${ }^{1 *}$ and A. Umamakeswari ${ }^{2}$ \\ 'Advanced Computing, SASTRA University, Tamil Nadu, India; ksruthianu@gmail.com \\ ${ }^{2}$ Department of Computer Science and Engineering, SASTRA University, Tamil Nadu, \\ India; aum@cse.sastra.edu
}

\begin{abstract}
In Wireless Sensor Network (WSN) each node must report to the Base Station (BS) with the frequency that the BS demands $\left(\mathrm{N}_{\mathrm{re}}\right)$. The paper proposes Enhanced Link aware Data Aggregation Mechanism (LDAM), an energy efficient data gathering algorithm that elects the Cluster Head (CH) and Gateway (GW) nodes based on the Predicted Transmission Count (PTC) that depends on the residual energy, forward and reverse delivery ratio. Once the clusters are formed, nodes calculate their distance(d) from $\mathrm{CH}$ based on which the nodes take-in varied transmission power. Based on the nodes weight, $\mathrm{CH}$ and GW are re-elected periodically and this enhances the nodes lifetime. Algorithm is simulated with varied number of nodes in the network, results prove to be efficient than LCM algorithm with respect to delivery ratio, energy consumed and average delay.
\end{abstract}

Keywords: Forward and Reverse Delivery Ratio, Link Aware, Varied Transmission Power

\section{Introduction}

Today, Wireless Sensor Network (WSN) is the most developing and challenging area of research, because of their real time application. Some of the applications include temperature monitoring system, war field mapping system, creating target aware homes etc. WSN is widely used in all these areas owing to its larger advantages and ease of access. Though there are number of advantages, a number of disadvantages are yet to be overcome. There are chances for the nodes to die due to smaller battery as well as high energy consumption for the task. This results in breakage of the chain communication wherein the nodes die continuously, which finally makes the network fail. So energy efficiency becomes a critical issue in WSN. After a lot of research, there came a solution called hierarchical clustering. Hierarchical clustering reduces the number of communication between the nodes. Components of hierarchical clustering are:
1. Sensor Nodes: Interior components that are used in WSN. They perform all important and basic task of the network: Sensing, Computing and Communicating.

2. Clusters: Organizational structures of WSN. Dense nodes that are deployed in the required environment are broken into simple groups called clusters for network management purpose.

3. Cluster Heads $(\mathrm{CH})$ : Organizational heads of the clusters, responsible for management, maintenance and routing in a cluster.

4. Gateway $(\mathrm{GW})$ : Nodes that are capable to hear two or more $\mathrm{CH}$.

5. Base Station (BS): Upper most level in the hierarchical structure of WSN. They provide a link between the network and end user.

$\mathrm{CH}$ are accountable for aggregating data from nodes in the respective clusters and then communicate to the BS.GW nodes become the only means for $\mathrm{CH}$ to

${ }^{*}$ Author for correspondence 
communicate. Choosing an effective node as $\mathrm{CH}$ and $\mathrm{GW}$ is regarded as an important challenge in hierarchical clustering.

Therefore selecting an efficient $\mathrm{CH}$ greatly enhances a reliable routing path and data delivery. With regards to choosing $\mathrm{CH}$ and $\mathrm{GW}$ there exist number of active clustering based algorithm but the communication overheads occurs in the particular kind of clustering. Further fixed transmission power leads to considerable reduction in the throughput. Therefore variable transmission power avoids energy wastage and enhances throughput.

The paper proposes Enhanced Link aware Clustering Mechanism (LDAM), a data aggregation algorithm based on passive clustering technique that concentrates both on the efficient $\mathrm{CH}$ and GW selection process and varied transmission power during the data aggregation process. Passive clustering concentrates on the link condition and state of the nodes. Based on the state of the sender and receiver nodes, nodes decide whether there must be a state change or not.

\section{Literature Survey}

There exist a lot of hierarchical clustering algorithms that deal with energy efficiency of nodes. In the Lowest ID Clustering algorithm (LIC) ${ }^{3}$, nodes have a unique ID. It chooses a node with least possible ID as $\mathrm{CH}$ when compared to the other nodes in the cluster. Periodically the nodes broadcast the list of nodes it can hear. When the nodes receive the list, node ID is compared and the node that hears to the nodes with higher node ID is declared as the $\mathrm{CH}$. This concludes that $\mathrm{CH}$ of the cluster has the lowest node ID than all the other nodes in the particular cluster. This is the simplest method, whereas the disadvantage is that some incapable nodes are prone to die due to energy drain.

Highest Connectivity Clustering algorithm $(\mathrm{HCC})^{4}$, is based on communication range of the nodes. Nodes periodically communicate with the nodes that are in the communication range. Node with highest node connectivity is selected as the $\mathrm{CH}$. There are chances for the $\mathrm{CH}$ to vary if at least any one of the node moves from the communication range of the nodes. Periodically the highest degree is measured to check the local highest node degree. Re-clustering becomes possible when two $\mathrm{CHs}$ move into a communicating range which may reduce the throughput. The main reason for this disadvantage is the unlimited number of nodes in a cluster. There exists an algorithm that overcomes these disadvantages.

In K-Hop Connectivity algorithm ${ }^{5}$, the same metrics of the previously mentioned algorithms are adopted. This algorithm overcomes the drawbacks of LIC and HCC, considering both the node ID and communication range of the node by setting Upper bound (U) and Lower bound (L) for number of nodes that can exist in a cluster. Number of $\mathrm{CH}$ formed is based on the $\mathrm{U}$ and $\mathrm{L}$. When the number of nodes is less than $\mathrm{L}$, nodes combine with the nearby clusters and if the contrary occurs, the clusters are split into two. All these algorithms are based on the concept called active clustering, where each node sends constant control packets to all the neighbouring nodes.

Proposed algorithm is based on Passive clustering ${ }^{6}$. In passive clustering there exist external and internal states of the nodes. This kind of clustering does not use control packets, nodes communicate via the packets that communicate. Reduced control packets reduce the overheads and increase the throughput. $\mathrm{CHs}$ are elected based on their states. Link Aware Clustering Mechanism is a passive clustering based algorithm, that selects the $\mathrm{CH}$ based on Predicted Transmission Count (PTC) ${ }^{1}$, that is extracted from Expected Transmission Count (ETC). PTC depends on nodes residual energy, distance between nodes and the nodes forward and reverse ratio. The major disadvantage of the algorithm is that the selected single node remains the $\mathrm{CH}$ for the entire network operation.

There exist some more hierarchical protocols that concentrate on energy efficiency. One such algorithm is Low Energy Adaptive Clustering Hierarchy (LEACH) algorithm ${ }^{7}$. The main aim of the algorithm is to form the clusters based on the Received Signal Strength Indicator (RSSI) and use $\mathrm{CH}$ for aggregating data and communicating it to the base station. The main disadvantage of LEACH is it selects $\mathrm{CH}$ in a random manner.

Hybrid Energy Efficient Distributed (HEED) algorithm $^{8}$, is an extended version of LEACH which takes into account the nodes residual energy and inter communication speed. The disadvantage of HEED is the multiple control messages transferred between nodes which increases the communication overheads.

Distributed Efficient Clustering Algorithm (DECA) ${ }^{9}$ is an enhancement of HEED, that considers residual energy, nodes unique ID and connectivity parameters. This further enhances the nodes lifetime but still there exist some drawbacks they are: fixed transmission power, fixed $\mathrm{CH}$ for the entire nodes lifetime. 
To overcome the disadvantages of LCM and DECA, an Enhanced Link Aware Data Aggregation Mechanism (LDAM) is proposed where both on weight based $\mathrm{CH}$ change and variable energy during the data aggregation phase.

\section{Proposed System}

LDAM is a complete algorithm, which includes both the $\mathrm{CH}$ selection and data aggregation thereafter communicates to the BS. The paper proposes the algorithm in two phases:

1. $\mathrm{CH}$ setup phase

2. Cluster maintenance phase

\subsection{CH Setup Phase}

Identifying the most efficient node to be the $\mathrm{CH}$ and $\mathrm{GW}$ will greatly reduce the energy consumption of the node. Every node that communicates with BS must report with the frequency $\left(\mathrm{N}_{\mathrm{re}}\right)$ that $\mathrm{BS}$ requires.

\subsubsection{Passive Clustering}

To identify the node, the proposed system is based on the passive clustering technique. With respect to passive clustering, clustering nodes may exist in different states:

a) External States: Initial (IN), Ordinary (OD), Distributed GW (D_GW, CH and GW

b) Internal States: GW_Ready (GW_R), ) CH_Ready ( $\left.\mathrm{CH} \_\mathrm{R}\right)$.

Internal states, representing the tentative state of the node, sends out a data packet and enter the external state. External Nodes receive the data packet and enter into the internal state. GW_R and CH_R state are only capable to become the GW and $\mathrm{CH}$ nodes. $\mathrm{CH}$ and $\mathrm{GW}$ selection is based on the PTC value of the nodes.

Passive clustering uses two mechanisms to elect $\mathrm{CH}$ and GW, the Declaration Wins rule and Gateway Heuristic.

1. First Declaration Wins rule: Nodes that are capable to become $\mathrm{CH}$ and $\mathrm{GW}$, first claims themselves as the $\mathrm{CH}$ or GW. Nodes that claim first are elected as $\mathrm{CH}$ or GW.

2. Gateway Heuristic: Mechanism determines the minimum number of GW required maintaining the network connectivity.

\subsubsection{Predicted Transmission Count (PTC:}

PTC checks node capability to have persistent communication with the neighbours. Proposed PTC considers the link condition and node status, to evaluate the priority of the capable nodes to be elected as the CH or GW. Link condition is selected as the parameter because, once the link between nodes is strong there are less chances for the chain to break, this controls retransmission. So the candidate linked with strong link condition is preferred to be $\mathrm{CH}$ or $\mathrm{GW}$ nodes. PTC value is derived from ETC ${ }^{10,11}$ which regularly measures the nodes forward and the reverse ratio between nodes.

$\operatorname{PTC}[\mathrm{p}][\mathrm{q}]=\operatorname{Res} /\left(\left(\operatorname{ETC}_{\mathrm{p}}[\mathrm{p}][\mathrm{q}]{ }^{*} \mathrm{E}_{\mathrm{tx}}(\mathrm{k}\right.\right.$, dist $\left.)\right)$

$\mathrm{E}_{\mathrm{tx}}(\mathrm{k}, \mathrm{dist})$ : Distance between nodes

ETC $_{\mathrm{p}}[\mathrm{p}][\mathrm{q}]$ : Expected Transmission Count

There are two types in first order model, Multipath model $^{12}$ and free space model ${ }^{13}$. When the distance between the communicating nodes in greater than the threshold value, then the system uses multipath channel model, else it uses free channel space model.

$$
\begin{aligned}
& \operatorname{ETC}[\mathrm{p}][\mathrm{q}]=1 /\left(\mathrm{E}_{\mathrm{f}}[\mathrm{p}][\mathrm{q}]^{*} \mathrm{E}_{\mathrm{r}}[\mathrm{p}][\mathrm{q}]\right) \\
& \operatorname{Etx}(k \cdot d i s t)= \begin{cases}\mathrm{k}^{*} \mathrm{E}_{\text {elc }}+\mathrm{k} \cdot \in_{\mathrm{fs}} \cdot d[\mathrm{p}][\mathrm{q}]^{2}, & \operatorname{dist}[\mathrm{p}][\mathrm{q}]<\mathrm{d}_{0} \\
\mathrm{k}^{*} \mathrm{E}_{\text {elc }}+\mathrm{k} \cdot \in_{\mathrm{ms}} \cdot d[\mathrm{p}][\mathrm{q}]^{4}, & \operatorname{dist}[\mathrm{p}][\mathrm{q}]>=\mathrm{d}_{0}\end{cases}
\end{aligned}
$$

Nodes constantly measure the Forward delivery ratio $\left(\mathrm{E}_{\mathrm{f}}[\mathrm{i}][\mathrm{j}]\right)$ and Reverse delivery ratio $\left(\mathrm{E}_{\mathrm{r}}[\mathrm{p}][\mathrm{q}]\right)$, which is further used to find the ETC value.

Steps to be performed in $\mathrm{CH}$ selection phase:

1. State transition in node

2. Calculate the priority value

3. Determine the $\mathrm{CH}$ and $\mathrm{GW}$ of nodes.

\subsubsection{State Transition in nodes}

State transmission in nodes occurs when internal nodes transmit a packet and external state nodes receive that packet. Algorithm for this state transmission is mentioned below. $\mathrm{ST}_{\text {cur }}$ and $\mathrm{ST}_{\text {new }}$ represent the current and the new state of the nodes respectively.

\footnotetext{
Input: $\mathrm{ST}_{\text {cur }}[\mathrm{p}], \mathrm{ST}_{\text {cur }}[\mathrm{q}], \mathrm{ID}[\mathrm{q}]$

if (node != BS)

\{

case IN:
}

Algorithm 1:To perform state transition for nodes: 


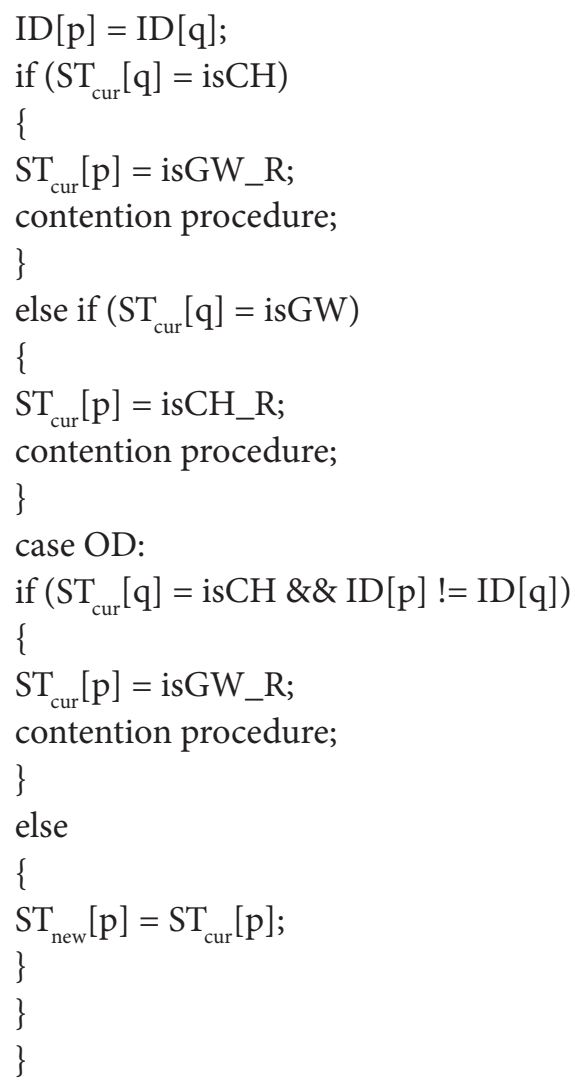

Algorithm can be explained in the following steps:

When the node receives a message from the sender, it does the following steps to know whether there must be a state change in the node. It's assumed that the node is in IN state in this case, and the steps are:

1. Checks if the sender is from the same cluster

2. Changes its ID to that of the sender

3. If the sender is $\mathrm{CH}$ then state changes to $\mathrm{GW} \_\mathrm{R}$

4. Else if the sender is GW then state transmits to $\mathrm{CH}$ _R

5. Then node enters the Contention procedure (Algorithm 2) to calculate the priority and elect $\mathrm{CH}$ and GW.

6. Once the node is elected, they start forwarding the messages received.

\subsubsection{Contention Procedure}

Neighbouring nodes enter into the contention procedure to identify the best node to be elected among them.

\footnotetext{
Algorithm 2: Calculate

$\Theta \mathrm{P}_{\mathrm{i}}$ - highest probability value

$\mathrm{T}_{\mathrm{i}}$-Time slot the node must wait before the node must start the data communication.
}

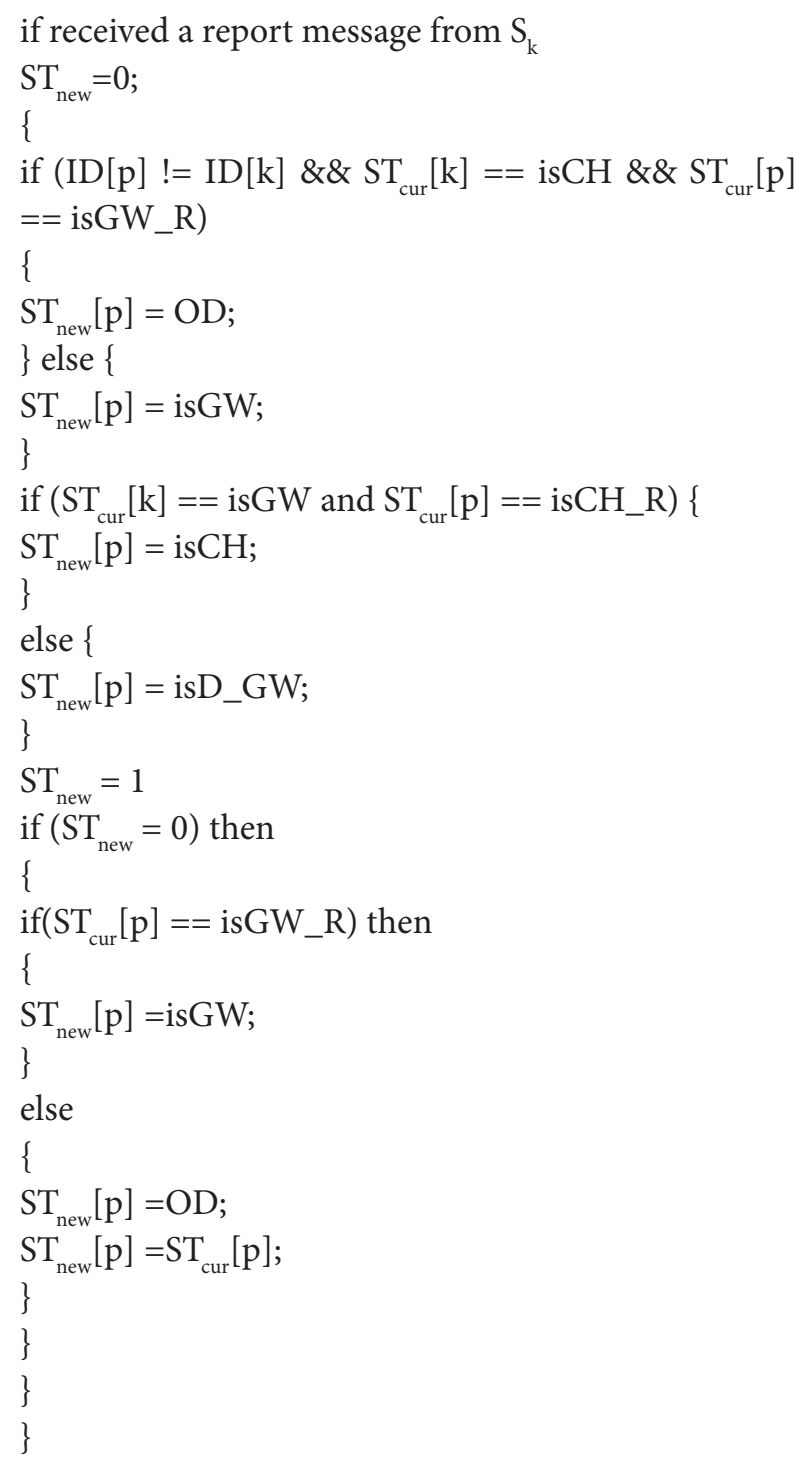

Algorithm 3: Calculate the Priority value: Complete the process for all CH_R and GW_R nodes. Input: Number of nodes neighbouring nodes and $\mathrm{N}_{\mathrm{re}}$ Output: $P_{i}$

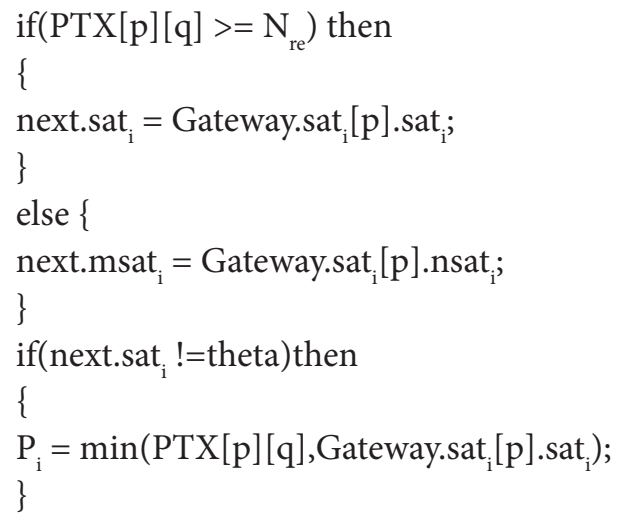




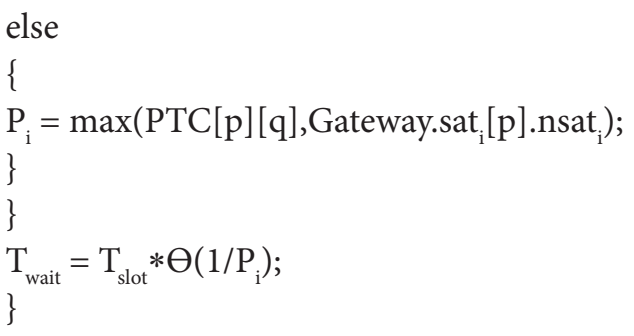

Algorithm can be explained in the following steps,

1. Calculate the PTC value for all the neighbouring nodes

2. Divide the neighbouring nodes into 2 subset as sat ${ }_{i}$ and nsat, subset of nodes that satisfy the $\mathrm{N}_{\mathrm{re}}$ value and nodes that does not satisfy respectively.

3. sat ${ }_{i}$ is empty then elect the maximum PTC value of nsat $_{i}$ as the $P_{i}$,else elect the minimum PTC of sat ${ }_{i}$ as the $P_{i}$ value.

4. Waiting time to carry out the random backoff method is calculated based on this $P_{i}$ value.

Node with the best $\mathrm{P}_{\mathrm{i}}$ value is selected as the $\mathrm{CH}$ and GW nodes. Once the nodes are selected, they start communicating in an hierarchical fashion.

\subsection{Cluster Maintenance Phase}

After the $\mathrm{CH}$ selection, nodes in the cluster tend to communicate with the $\mathrm{BS}$ via the $\mathrm{CH}$. Proposed algorithm carries out energy efficient intra cluster communication. Nodes to communicate data to their respective $\mathrm{CH}$, initially checks RSSI ${ }^{14-17}$ value from the previously received messages. Using this RSSI value, the distance between nodes and $\mathrm{CH}$ is estimated. The distance formula is

$d=10^{-(\mathrm{RSSI}+} \mathrm{S}_{\mathrm{rec}+\text { offset) } / 10 \mathrm{sp}}$

Based on the calculated distance ${ }^{18}$, node assign the transmitting power that is needed for the communication. Longer the distance, higher the transmission power. Otherwise the transmission energy assigned less for the nodes. "sp" is the signal propagation constant, $\mathrm{S}_{\text {rec }}$ is the received signal strength from nodes within one meter, offset is the random measured value ranging from 0 to $1 \mathrm{dBm}$. Varying the transmission power has two major advantages viz, reduces the energy consumed and increases the throughput.

Literature says, varied transmission power reduces the RSSI value in meshed network. The following points witness that varied transmission power ${ }^{19}$ increases the performance of the proposed algorithm.
1. Varied transmission power does not support the meshed network as the data transfer rate is higher in this case.

2. In the case of the proposed algorithm, transmission power is constant in inter cluster communication and the selection of $\mathrm{CH} \& \mathrm{GW}$. This kind of transmission occurs in the case of intra cluster communication.

3. In a network there exist number of clusters; in this case it is quite obvious that when all nodes use the same amount of energy, it surely affects the network performance.

So the proposed algorithm utilises varied transmission power in the data aggregating phase of the network. When the data reaches $\mathrm{CH}$, it performs data aggregation and transmits to BS.

As the process proceeds, nodes remain the $\mathrm{CH}$ and GW for a certain amount of time, which is estimated by the following parameters: Res and node ID. Using these parameters, nodes calculate their weight ${ }^{2}$. Estimated time slot for every node is inversely proportional to weight.

Time slot $=1 /$ weight

weight $=\alpha * \operatorname{Res}+\beta * \operatorname{ID}, 0<\alpha<\beta<1$

Time slot for a node is greatly dependent on the Res when compared to ID. In case a tie occurs, ID of the node acts as the tie breaker. Node with larger Res will last as $\mathrm{CH}$ or GW for shorter time slot. This also permits the energy conservation of the nodes.

\section{Performance Metrics}

This section defines the simulation environment, parameters, metrics measured and comparison with the existing algorithm. Study of the proposed algorithm is done in NS2, simulation results are compared with LCM to prove the efficiency of the proposed algorithm. Static nodes are randomly scattered with the constant initial energy for nodes. Table 1 shows the parameters used for testing the proposed algorithm.

Algorithm is tested for the following metrics:

1. Message Delivery Ratio: It is the total number transactions arriving at the BS to the total number of messages communicated by $\mathrm{CH}$. As proposed system supports hierarchical routing, nodes selected to reach the BS must be stable, else there are chances for the link to be broken and then reconstruction of network 
Table 1. Simulation Parameters and Values

\begin{tabular}{ll}
\hline Parameters & Values \\
\hline Size of the Network & $300 \mathrm{~m} \times 300 \mathrm{~m}$ \\
Number of Nodes & $50,100,150,200,250$ \\
Communication range & $50 \mathrm{~m}$ \\
Size of the packet & $2000 \mathrm{bits}$ \\
$\mathrm{E}_{\mathrm{elc}}$ & $50 \mathrm{~nJ} / \mathrm{bit}$ \\
$\in_{\mathrm{ms}}$ & $0.0013 \mathrm{pJ} / \mathrm{bit} / \mathrm{m}^{4}$ \\
$\in_{\mathrm{fs}}$ & $10 \mathrm{pJ} / \mathrm{bit} / \mathrm{m}^{2}$ \\
$\mathrm{~N}_{\mathrm{re}}$ & 5 \\
Simulation time & $100 \mathrm{~s}$ \\
\hline
\end{tabular}

must occur. This kind of chance is very much reduced with the proposed algorithm. Comparisons are made between LCM and LADM by increasing the number of nodes. LADM produces around $2.19 \%$ better message delivery than LCM (Figure 1).

2. Energy Consumed: is defined as the amount of energy spent by the node for communicating with respect to the initial energy that the node contained. Nodes consume more energy during the clustering phase than the maintenance phase. Only nodes that are in CH_R and GW_R are processed to be elected as $\mathrm{CH}$ and GW and the varied transmission power given to nodes in the maintenance phase further reduces the amount of energy consumed by the nodes. Comparative results show around $2.5 \%$ decrease in the energy consumed by the network using LDAM algorithm (Figure 2).

3. Average Delay: The time delay experienced by the sender before the destination node receives the transmitted packet. Generally nodes experience greater delay only when some of the nodes along the routing path as the continuation packets are to be retransmitted and energy loss may occur which in turn affects the life of the node. Test result shows around 3.8\% decrease in the delay when the proposed algorithm is used (Figure 3).

\section{Conclusion}

The proposed algorithm concentrates on residual energy and link condition to estimate the priority of nodes to be elected as the $\mathrm{CH}$ or $\mathrm{GW}$ nodes to guarantee reliable routing path, data delivery and packet delivery ratio.

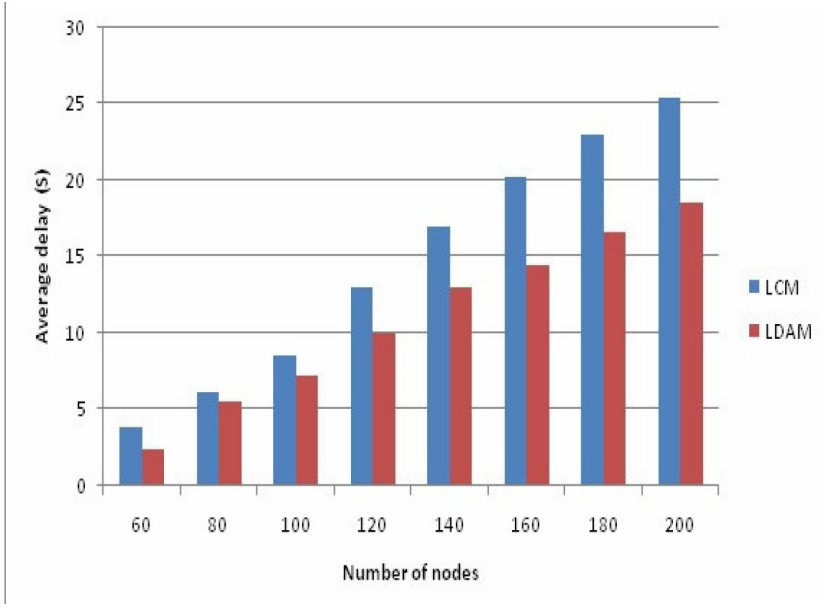

Figure 1. Message Delivery Ratio in LDAM.

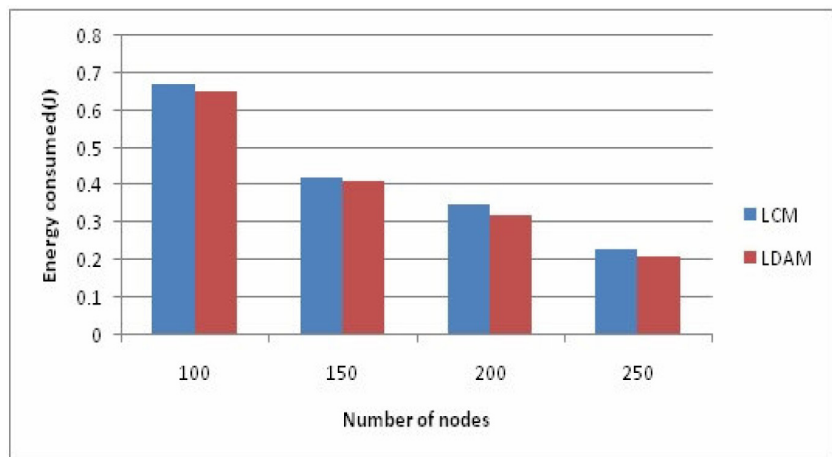

Figure 2. Energy Consumed in LDAM.

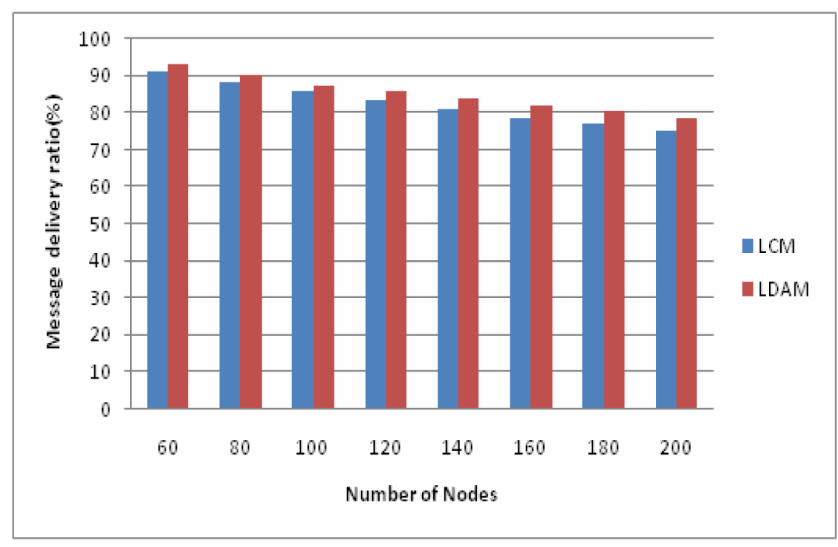

Figure 3. Average Message Delay in LDAM.

Variable transmission power supplied to nodes during the communication phase leads to the increase of lifetime and throughput. Simulation results clearly depicts that proposed LDAM with respect in average delay, energy consumption and message delivery ratio shows better results than LCM algorithm. 


\section{References}

1. Wang S-S, Chen Z-P. LCM: a link-aware clustering mechanism for energy-efficient routing in wireless sensor networks. IEEE Sensor J. 2013; 13(2).

2. Khan AR, Madani SA, Hayat K, Khan SU. Clustering-based power-controlled routing for mobile wireless sensor networks. Int J Comm Syst. 2011.

3. Gerla M, Tsai TJ. Multiuser, mobile, multimedia radio network. Wireless Network. 1:255-65

4. Tsigas P. Project on mobile ad hoc networking and clustering for the course EDA390 Computer communication and distributed systems. Manual for University Course.

5. Chen G, Nocetti FG, Gonzalez JS, Stojmenovic I. Connectivity based k-hop Clustering in wireless networks. Proceedings of the 35th Hawaii International Conference on System Sciences.

6. Kwon TJ, Gerla M. Efficient flooding with Passive Clustering (PC) in ad hoc networks. ACM SIGCOMM. Comput Commun Rev. 2002 Jan. 32(1):44-56.

7. Buczak VR, Jamalabad AL. Self-organization of a heterogeneous sensor network by genetic algorithms. Engineering Systems Through Artificial Neural Networks. 1998; 8:25964.

8. Younis O, Fahmy S. HEED: a hybrid, energy-efficient, distributed clustering approach for ad hoc sensor networks. IEEE Transactions on Mobile Computing. 2004; 3(3):36679.

9. Yu M, Li JH, Levy R. Mobility resistant clustering in multihop wireless networks. J Network. 2006; 1(1):12-19.

10. Couto DD, Aguayo D, Bicket J, Morris R. A high-throughput path metric for multi-hop wireless routing. Proc ACM Int Conf Mobile Comput Netw. 2003 Sep; 134-46.

11. Eric R, Jayesh S, Yogita AM, Qiu L. SOAR: Simple opportunistic adaptive routing protocol for wireless mesh networks. IEEE Transaction Mobile Computing. 2009 Dec; $8(12): 1622-35$

12. Heinzelman WB, Chandrakasan AP, Balakrishnan $\mathrm{H}$. An application-specific protocol architecture for wireless microsensor networks. IEEE Trans Wireless Commun. 2002 Oct; 1(4):660-70.

13. Rappaport TS. Wireless communications: principles and practice. Englewood Cliffs, NJ: Prentice-Hall; 2002.

14. Lau EEL, Lee BG, Lee SC, Chung WY. Enhanced RSSIbased high accuracy real-time user location tracking system for indoor and outdoor environments. International Journal on Smart Sensing and Intelligent Systems. 2008; 1(2):12-19.

15. Mao G, Fidan B, Anderson BDO. Wireless sensor network localization techniques. Computer Networks. 2007; 51:2529-53.

16. Barsocchi P, Lenzi S, Chessa S, Giunta G. Virtual calibration for RSSI-based indoor localization with IEEE 802.15.4. Proceedings of The IEEE International Conference on Communications; 2009; Dresden, Germany. p. 1-5.

17. Jeong S, Oh C. RSSI-based cooperative localization algorithm using virtual reference nodes. Proceedings of NetApps2008, International Conference on Network Applications, Protocols and Services, Utara, Malayasia; 2008.

18. Chen W, Meng X. A cooperative localization scheme for Zigbee-based wireless sensor networks. Proceedings of the 14th IEEE International Conference (ICON '06); 2006; MT, U.S.A.: Big Sky. p. 1-5.

19. Max S, Wang T. Transmit power control in wireless mesh networks considered harmful. Proceedings of, Second International Conference on Advances in Mesh Networks. 2009 Jun; Greece. p. 73-8. 